\title{
Leigh's disease: significance of the biochemical changes in brain ${ }^{1}$
}

\author{
JEROME V. MURPHY ${ }^{2}$ AND LINDA CRAIG
}

\begin{abstract}
From the Departments of Pediatrics and Neurology of the University of Pittsburgh School of
\end{abstract} Medicine and the Children's Hospital of Pittsburgh, Pittsburgh, Pennsylvania 15213, U.S.A.

SYNOPSIS Analysis of five brains from patients with Leigh's disease demonstrates an accumulation of thiamine pyrophosphate and a deficiency of thiamine triphosphate. The enzyme which converts thiamine pyrophosphate to thiamine triphosphate was normally active in two of these brains, suggesting that the inhibitor found in Leigh's disease is probably producing the observed neurochemical changes. Reasons for the histological similarity between Leigh's and Wernicke's diseases are suggested.

Leigh's disease, subacute necrotizing encephalomyelopathy, is a recessively inherited disorder afflicting children of all ages (Pincus, 1972). The clinical findings are nonspecific and the diagnosis is made by finding the typical pathological changes in the brain of the patient or the brain of a similarly afflicted sibling (Montpetit et al., 1971).

Two patients with Leigh's disease have had a deficiency of the enzyme, pyruvate carboxylase (Hommes et al., 1968; Tang et al., 1972), but this has not been a consistent feature in all patients with the disease (Grover et al., 1972; Murphy, 1974). A second biochemical feature of Leigh's disease is the presence of an inhibitor to the synthesis of thiamine triphosphate by brain (Cooper et al., 1969). This inhibitor can be

\footnotetext{
1 This research was supported by General Research Support grants from the National Institutes of Health to the Children's Hospital of Pittsburgh and to the University of Pittsburgh School of Medicine, and by a grant (HD 07465-01) from the Institute of Child Health and Human Development.

2 Present address: Milwaukee Children's Hospital, 1700 West Wisconsin Avenue, Milwaukee, Wisconsin 53233, U.S.A.

(Accepted 9 June 1975.)
}

detected in body fluids of untreated patients with Leigh's disease as well as in the urine of obligate carriers of the Leigh's disease gene (Murphy et al., 1974). Thiamine appears to inactivate the inhibitor, as treatment of either patients or carriers with large amounts of thiamine effectively reduces the activity of inhibitor in urine (Murphy, 1973; Murphy et al., 1974).

The reaction which is inhibited is the conversion of thiamine pyrophosphate and ATP to thiamine triphosphate and ADP by a phosphotransferase (Fig. 1a). Thiamine triphosphate is also synthesized by liver enzymes, but the inhibitor is specific for the brain enzyme and does not affect the hepatic synthesis of thiamine triphosphate (Murphy et al., 1974).

At issue is whether this inhibitor is a consequence of Leigh's disease or whether it has an aetiological rôle in producing the changes of the disease. If the latter be the case, at least four conditions must be met: (1) there should be an

(a) TPP + ATP $\stackrel{\text { ATP }- \text { thiamine diphosphate phosphokinase }}{\longrightarrow}$ TTP + ADP

(b) TTP $\underset{\text { Nucleoside triphosphate phosphatase }}{\longrightarrow} \mathrm{TPP}+\mathrm{Pi}$

FIG. 1 Reactions involved in the synthesis (a) and the hydrolysis (b) of TTP $(\mathrm{TPP}=$ thiamine pyrophosphate $. \mathrm{TTP}=$ thiamine triphosphate $)$. 
accumulation of the substrate of the inhibited reaction, thiamine pyrophosphate; (2) thiamine triphosphate, the end-product, should be present in reduced amounts; (3) the specific brain phosphotransferase should be present and normally active; and (4) the inhibitor should be present in the central nervous system.

In the present study, we have examined the first three conditions. The demonstration of inhibitor in the cerebrospinal fluid by Cooper et al. (1969) fulfils the fourth condition.

\section{METHODS}

MEASUREMENT OF THIAMINE AND ITS PHOSPHATE ESTERS One frontal pole of each of five brains subsequently diagnosed as Leigh's disease was frozen $\left(-20^{\circ} \mathrm{C}\right)$ at necropsy. Four patients had the inhibitor in their urine and the fifth patient was not previously studied for Leigh's disease. The pathological diagnoses of a control series of six brains included sudden infant death syndrome, congenital heart disease, and Werdnig-Hoffman disease. In all 11 brains the opposite frontal pole was histologically normal. Generous portions of liver from three patients with Leigh's disease and four controls were similarly frozen at necropsy for thiamine studies.

After variable periods of frozen storage the tissues were thawed and homogenized in water, $1 \mathrm{~g} / 7 \mathrm{ml}$. Five millilitres of $10 \%$ trichloroacetic acid (TCA) was added to $20 \mathrm{ml}$ of this homogenate, mixed and centrifuged (2000 $\mathrm{g}$ for $20 \mathrm{~min}$ ). After four extractions with equal amounts of ether, the TCA-free aqueous extract was concentrated to $0.4-0.5 \mathrm{ml}$. Thiamine, thiamine monophosphate, thiamine pyrophosphate, and thiamine triphosphate concentrations were determined by the electrophoretic method of Itokawa and Cooper (1970a).

CEREBRAL THIAMINE TRIPHOSPHATE SYNTHESIS Large amounts of a cerebral hemisphere from each of two brains of patients with Leigh's disease, the brain of a patient with congenital heart disease, and a bovine brain were obtained within three hours of death. An acetone precipitate of an aqueous extract of these brains was assayed for phosphotransferase activity by a previously described method (Murphy et al., 1974). The Lowry method was used to measure the soluble protein content of this acetone precipitate (Lowry et al., 1951).

THIAMINE TRIPHOSPHATASE The conversion of thiamine triphosphate to thiamine pyrophosphate was studied under the same conditions as the synthesis of thiamine triphosphate with two minor modifications: the reaction mixture contained no ATP and $5 \mu \mathrm{g}$ thiamine triphosphate replaced the thiamine pyrophosphate. Water replaced each reagent in the appropriate controls and the reaction mixture was incubated and assayed as previously described (Murphy et al., 1974).

\section{RESULTS}

CONCENTRATION OF THIAMINE AND ITS PHOSPHATE ESTERS 1. Brain (Fig. 2) There was no difference in the thiamine and thiamine monophosphate concentrations in children with Leigh's disease or controls $(P<0.4$ and $\mathbf{P}<0.2$, respectively). The thiamine pyrophosphate values were higher in Leigh's disease brains, despite some

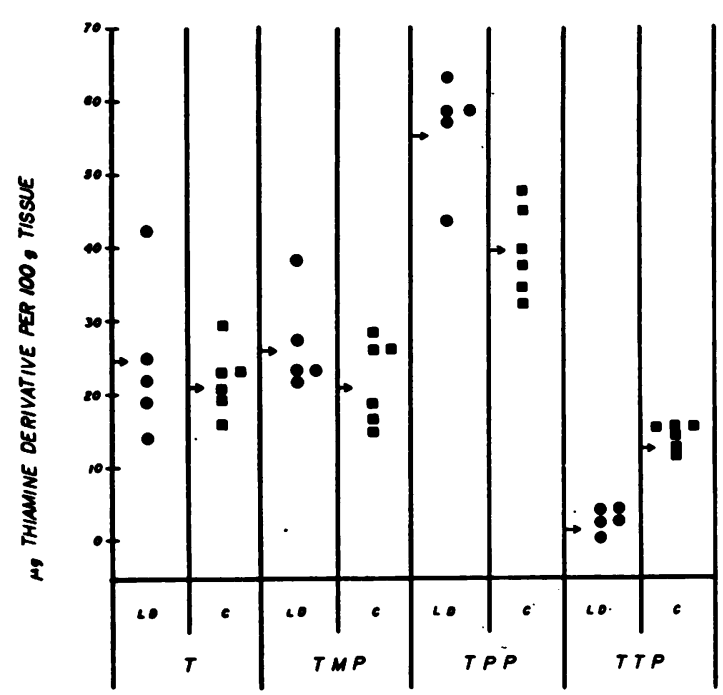

FIG. 2 Concentrations of thiamine and its phosphate esters in Leigh's disease (LD) and control (C) brains. Each point is the average of duplicate determinations on the same brain and the arrows denote the mean value for each series $(\mathrm{T}=$ thiamine, $\mathrm{TMP}=$ thiamine monophosphate, $\mathrm{TPP}=$ thiamine pyrophosphate, and TTP = thiamine triphosphate).

overlap between the two series $(\mathrm{P}<0.005)$. There was a distinct reduction in the thiamine triphosphate concentrations of the Leigh's disease brains as compared to controls $(\mathrm{P}<0.001)$.

2. Liver (Table 1) Thiamine, thiamine monophosphate, thiamine pyrophosphate, and thia- 
TABLE 1

THIAMINE PYROPHOSPHATE AND THIAMINE TRIPHOSPHATE CONCENTRATIONS IN LIVER OF FOUR CONTROLS AND THREE PATIENTS WITH LEIGH'S DISEASE*

\begin{tabular}{lcc}
\hline Source of liver & $\begin{array}{c}\text { Thiamine pyrophosphate } \\
(\mu \mathrm{g} / 100 \mathrm{~g})\end{array}$ & $\begin{array}{c}\text { Thiamine triphosphate } \\
(\mu \mathrm{g} / 100 \mathrm{~g})\end{array}$ \\
\hline Leigh's disease & $\begin{array}{c}83.9 \\
(54.1-135.4) \\
91.0\end{array}$ & $\begin{array}{c}38.1 \\
(17.2-74.8) \\
33.4\end{array}$ \\
Control & $(60.7-130)$ & $(23.5-38.0)$ \\
& & \\
\hline
\end{tabular}

* The results are expressed as micrograms of thiamine ester per $100 \mathrm{~g}$ tissue. The range of values is in parentheses below the mean.

mine triphosphate concentrations were the same in the livers of three patients with Leigh's disease and four control patients (Table 1).

Cerebral thiamine synthesis The ability of the acetone precipitate of two brains from children with Leigh's disease to synthesize thiamine triphosphate did not differ significantly from controls. The phosphotransferase activity, expressed as micrograms of thiamine triphosphate synthesized/mg protein/hour, was 1.50 and 1.38 in the Leigh's patients compared with values of 1.55 and 1.48 in the control and bovine brains. (We have observed that acetone inactivates the inhibitor in such a preparation.)

Thiamine triphosphatase When the thiamine triphosphate was incubated with the acetone extract of bovine brain a small amount of the thiamine triphosphate $(2.3 \mu \mathrm{g} / \mathrm{ml})$ was converted to thiamine pyrophosphate. This conversion of

TABLE 2

THIAMINE TRIPHOSPHATASE ACTIVITY OF INHIBITOR*

\begin{tabular}{lcccc}
\hline & \multicolumn{4}{c}{ Conditions of reaction } \\
\cline { 2 - 5 } & TTP alone & TTP+ urinet TTP+acetone $\begin{array}{c}\text { TTP + urine† } \\
\text { extract } \\
\text { +acetone } \\
\text { extract }\end{array}$ \\
\hline $\operatorname{TTP}(\mu \mathrm{g} / \mathrm{ml})$ & 11.4 & 11.5 & 9.1 & 9.9 \\
\hline
\end{tabular}

* Thiamine triphosphate (TTP) was incubated with patient urine and a microsomal extract of bovine brain to see if the 'inhibitor' worked by hydrolysing thiamine triphosphate (TTP $=$ thiamine triphosphate). + This urine inhibited the cerebral phosphotransferase $48 \%$ (Murphy et al., 1974). thiamine triphosphate to thiamine pyrophosphate was not influenced by the presence of inhibitor (Table 2).

\section{DISCUSSION}

These observations confirm that the brains of patients with Leigh's disease are deficient in thiamine triphosphate (Cooper et al., 1969; Cooper and Pincus, 1972) and demonstrate for the first time that these same brains have increased amounts of its precursor thiamine pyrophosphate (Fig. 2). The fact that the opposite frontal pole of all the brains studied was histologically normal means that these changes were not artefacts of tissue destruction. Some of the thiamine triphosphate could have been converted to other products during the interval between death and necropsy, but the consistent difference between Leigh's disease and control brains indicates that the thiamine triphosphate is relatively reduced in the Leigh's disease brains.

There are at least three ways to explain the deficiency of thiamine triphosphate and the accumulation of thiamine pyrophosphate: (1) the enzyme converting thiamine pyrophosphate to thiamine triphosphate (Fig. 1a) could be inactive; (2) if the enzyme is active, an inhibitor could be blocking its action; or (3) thiamineo triphosphate could be converted to thiamine? pyrophosphate at an unusually rapid rate-that is, the reverse reaction of Fig. 1a. The first possibility, an enzyme deficiency, was studied by examining the phosphotransferase activity of the acetone extract of two Leigh's disease brains (see Results section, p. 1102.) According to this study, the phosphotransferase which synthesizes thiamine triphosphate is present and active in the two Leigh's disease brains studied.

The second explanation is more tenable, as an inhibitor to the cerebral synthesis of thiamine triphosphate has been described (Cooper et al., 1969; Murphy et al., 1974). The presence of this inhibitor in all body fluids, including cerebrospinal fluid (Cooper et al., 1969), indicates that it could limit the synthesis of thiamine triphosphate in the brains of Leigh's disease patients.

The third explanation cited above suggests that this inhibitor, instead of limiting the synthesis of thiamine triphosphate, could be 
activating the conversion of thiamine triphosphate to thiamine pyrophosphate. Such an accelerated conversion would give the same changes in the concentrations of thiamine phosphate esters in Leigh's disease brain as seen in Fig. 2. This alternative hypothesis was evaluated by observing the conversion of thiamine triphosphate to thiamine pyrophosphate in the presence and absence of an inhibiting urine (Table 2). As the inhibitor did not accelerate the hydrolysis of thiamine triphosphate under these assay conditions, we assume the inhibitor works by blocking the synthesis of thiamine triphosphate (Fig. 1a). We have studied the hydrolysis of thiamine triphosphate on additional occasions and TTP hydrolysis was never accelerated in the presence of an inhibiting urine. It is still possible that the enzyme converting thiamine triphosphate to thiamine pyrophosphate, the reverse reaction of Fig. 1, is excessively active for other reasons and that this excessive breakdown of cerebral thiamine triphosphate contributes to the changes illustrated in Fig. 2.

Reviewing the data cited above we believe: (1) that the deficiency of thiamine triphosphate and the accumulation of thiamine pyrophosphate in the brains of Leigh's disease patients is best explained by the action of the inhibitor which is found in the body fluids of our patients with Leigh's disease, and (2) that the four conditions set forth in the introduction to support the rôle of the inhibitor in Leigh's disease have been fulfilled. These experimental data strongly support the aetiological rôle of the inhibitor in Leigh's disease, although not proving that this is the only biochemical aetiology of Leigh's disease.

That the deficiency of thiamine triphosphate could contribute to the histological changes of Leigh's disease is suggested by considering the changes in the brains of patients with Wernicke's disease. Both Leigh's and Wernicke's diseases have a similar histopathology despite regional differences (Montpetit et al., 1971). Wernicke's disease is associated with a deficiency of thiamine and presumably all thiamine esters, whereas Leigh's disease has a deficiency only of thiamine triphosphate. The inhibitor is absent in Wernicke's disease (Cooper and Pincus, 1972) and present in Leigh's disease. As both condi- tions have a deficiency of thiamine triphosphate in common, it is possible that this lack of thiamine triphosphate in brain produces the small symmetrical areas of capillary infiltration and necrosis in the base of the brain. It is possible that other unrecognized biochemical similarities could also contribute to the similar pathology. If a deficiency of thiamine triphosphate is producing some of the histological changes, thiamine triphosphate must have a vital rôle in cerebral metabolism (Itokawa and Cooper, 1970b).

We are grateful to $\mathrm{R}$. Glew for biochemical advice, to T. K. Oliver, Jr and J. Moossy for their helpful criticisms, to H. Carricato for her technical skills, to B. Willett for her secretarial assistance, and to T. Yusa for the gift of thiamine triphosphate.

\section{REFERENCES}

Cooper, J. R., Itokawa, Y., and Pincus, J. H. (1969). Thiamine triphosphate deficiency in subacute necrotizing encephalomyelopathy. Science, 164, 74-75.

Cooper, J. R., and Pincus, J. H. (1972). Role of thiamine triphosphate in subacute necrotizing encephalomyelopathy. Journal of Agriculture and Food Chemistry, 20, 490-493.

Grover, W. D., Auerbach, V. H., and Patel, M. S. (1972). Biochemical studies in subacute necrotizing encephalomyelopathy (Leigh's syndrome). Journal of Pediatrics, 81, 39-44.

Hommes, F. A., Polman, H. A., and Reerink, J. D. (1968). Leigh's encephalomyelopathy: an inborn error of gluconeogenesis. Archives of Disease in Childhood, 43, 423-426.

Itokawa, Y., and Cooper, J. R. (1970a). Electrophoretic separation and fluorometric determination of thiamine and its thiamine phosphate esters. In Methods of Enzymology, vol 18a, pp. 91-92. Academic Press: New York.

Itokawa, Y., and Cooper, J. R. (1970b). Ion movements and thiamine. 2. The release of the vitamin from membrane fragments. Biochimica Biophysica Acta, 196, 274-284.

Lowry, O. H., Rosebrough, N. J., Farr, A. L., and Randall, R. J. (1951). Protein determinations with folin phenol reagent. Journal of Biological Chemistry, 193, 265-275.

Montpetit, V. J. A., Andermann, F., Carpenter, S., Fawcett, J. S., Zborowska-Sluis, D., and Giberson, H. R. (1971). Subacute necrotizing encephalomyelopathy, a review and a study of two families. Brain, 94, 1-30.

Murphy, J. V. (1973). Subacute necrotizing encephalomyelopathy (Leigh's disease): detection of the heterozygous carrier state. Pediatrics, 51, 710-715.

Murphy, J. V. (1974). Efficacy of recommended therapeutic regimens in Leigh's disease. Developmental Medicine and Child Neurology, 16, 362-364.

Murphy, J. V., Craig, L.. J., and Glew, R. H. (1974). Leigh disease. Biochemical characteristics of the inhibitor. Archives of Neurology, 31, 220-227.

Pincus, J. H. (1972). Subacute necrotizing encephalomyelopathy (Leigh's disease): a consideration of clinical features and etiology. Developmental Medicine and Child Neurology, 14, 87-101.

Tang, T. T., Good, T. A., Dyken, P. R., Johnsen, S. D., McCreadie, S. R., Sy, S. T., Lardy, H. A., and Rudolph, F. B. (1972). Pathogenesis of Leigh's encephalomyelopathy. Journal of Pediatrics, 81, 189-190. 